Proceedings of the SIGLEX/SENSEVAL Workshop on Word Sense

Disambiguation: Recent Successes and Future Directions, Philadelphia,

July 2002, pp. 40-46. Association for Computational Linguistics.

\title{
Assessing System Agreement and Instance Difficulty in the Lexical Sample Tasks of SENSEVAL-2
}

\author{
Ted Pedersen \\ Department of Computer Science \\ University of Minnesota \\ Duluth, MN, 55812 USA \\ tpederse@d.umn.edu
}

\begin{abstract}
This paper presents a comparative evaluation among the systems that participated in the Spanish and English lexical sample tasks of SENSEVAL-2. The focus is on pairwise comparisons among systems to assess the degree to which they agree, and on measuring the difficulty of the test instances included in these tasks.
\end{abstract}

\section{Introduction}

This paper presents a post-mortem analysis of the English and Spanish lexical sample tasks of Senseval-2. Two closely related questions are considered. First, to what extent did the competing systems agree? Did systems tend to be redundant and have success with many of the same test instances, or were they complementary and able to disambiguate different portions of the instance space? Second, how much did the difficulty of the test instances vary? Are there test instances that proved unusually difficult to disambiguate relative to other instances?

We address the first question via a series of pairwise comparisons among the participating systems that measures their agreement via the kappa statistic. We also introduce a simple measure of the degree to which systems are complementary called optimal combination. We analyze the second question by rating the difficulty of test instances relative to the number of systems that were able to disambiguate them correctly.
Nearly all systems that received official scores in the Spanish and English lexical sample tasks of SEnSEVAL-2 are included in this study. There are 23 systems included from the English lexical sample task and eight from the Spanish. Table 1 lists the systems and shows the number of test instances that each disambiguated correctly, both by part of speech and in total.

\section{Pairwise System Agreement}

Assessing agreement among systems sheds light on whether their combined performance is potentially more accurate than that of any of the individual systems. If several systems are largely in agreement, then there is little benefit in combining them since they are redundant and will simply reinforce one another. However, if some systems disambiguate instances that others do not, then they are complementary and it may be possible to combine them to take advantage of the different strengths of each system to improve overall accuracy.

The kappa statistic (Cohen, 1960) is a measure of agreement between multiple systems (or judges) that is scaled by the agreement that would be expected just by chance. A value of 1.00 suggests complete agreement, while 0.00 indicates pure chance agreement. Negative values indicate agreement less than what would be expected by chance. (Krippendorf, 1980) points out that it is difficult to specify a particular value of kappa as being generally indicative of agreement. As such we simply use kappa as a tool for comparison and relative ranking. A detailed discussion on the use of kappa in natural language processing is presented in (Carletta, 1996). 
Table 1: Lexical Sample Systems

\begin{tabular}{|c|c|c|c|c|}
\hline system & \multicolumn{4}{|c|}{ correct instances } \\
\hline name & noun & verb & adj & total $(\%)$ \\
\hline \multicolumn{5}{|c|}{ English } \\
\hline & 1754 & 1806 & 768 & $4328(1.00)$ \\
\hline jhu_final & 1196 & 1022 & 562 & $2780(0.64)$ \\
\hline smuls & 1219 & 1016 & 528 & $2763(0.64)$ \\
\hline kunlp & 1171 & 1040 & 513 & $2724(0.63)$ \\
\hline $\operatorname{cs} 224 n$ & 1198 & 945 & 527 & $2670(0.62)$ \\
\hline lia & 1177 & 966 & 510 & $2653(0.61)$ \\
\hline talp & 1149 & 927 & 495 & $2571(0.59)$ \\
\hline duluth3 & 1137 & 840 & 497 & $2473(0.57)$ \\
\hline umcp & 1081 & 891 & 487 & $2459(0.57)$ \\
\hline ehu_all & 1069 & 891 & 480 & $2440(0.56)$ \\
\hline duluth4 & 1065 & 806 & 476 & $2346(0.54)$ \\
\hline duluth2 & 1056 & 795 & 483 & $2334(0.54)$ \\
\hline lesk_corp & 960 & 804 & 454 & $2218(0.51)$ \\
\hline duluthB & 1004 & 729 & 467 & $2200(0.51)$ \\
\hline uned_ls_t & 987 & 699 & 469 & $2155(0.50)$ \\
\hline common & 880 & 728 & 453 & $2061(0.48)$ \\
\hline alicante & 427 & 866 & 486 & $1779(0.41)$ \\
\hline uned_ls_u & 781 & 519 & 437 & $1736(0.40)$ \\
\hline clr_ls & 602 & 393 & 272 & $1267(0.29)$ \\
\hline iit2 & 541 & 348 & 166 & $1054(0.24)$ \\
\hline iit1 & 516 & 337 & 182 & $1034(0.24)$ \\
\hline lesk & 467 & 328 & 182 & $977(0.23)$ \\
\hline lesk_def & 438 & 159 & 108 & $704(0.16)$ \\
\hline random & 303 & 153 & 155 & $611(0.14)$ \\
\hline \multicolumn{5}{|c|}{ Spanish } \\
\hline & 799 & 745 & 681 & $2225(1.00)$ \\
\hline jhu & 560 & 478 & 546 & $1584(0.71)$ \\
\hline $\operatorname{cs} 224$ & 520 & 443 & 526 & $1489(0.67)$ \\
\hline umcp & 482 & 435 & 479 & $1396(0.63)$ \\
\hline duluth8 & 494 & 382 & 494 & $1369(0.62)$ \\
\hline duluth7 & 470 & 374 & 480 & $1324(0.60)$ \\
\hline duluth9 & 445 & 359 & 446 & $1250(0.56)$ \\
\hline duluthY & 411 & 325 & 434 & $1170(0.53)$ \\
\hline alicante & 269 & 381 & 468 & $1118(0.50)$ \\
\hline
\end{tabular}

To study agreement we have made a series of pairwise comparisons among the systems included in the English and Spanish lexical sample tasks. Each pairwise combination is represented in a $2 \times 2$ contingency table, where one cell represents the number of test instances that both systems disambiguate correctly, one cell represents the number of instances where both systems are incorrect, and there are two cells to represent the counts when only one system is correct. Agreement does not imply accuracy, since two systems may get a large number of the same instances incorrect and have a high rate of agreement.

Tables 2 and 3 show the system pairs in the English and Spanish lexical sample tasks that exhibit the highest level of agreement according to the kappa statistic. The values in the both-one-zero column indicate the percentage of instances where both systems are correct, where only one is correct, and where neither is correct. The top 15 pairs are shown for nouns and verbs, and the top 10 for adjectives. A complete list would include about 250 pairs for each part of speech for English and 24 such pairs for Spanish.

The utility of kappa agreement is confirmed in that system pairs known to be very similar have correspondingly high measures. In Table 2 , duluth2 and duluth3 exhibit a high kappa value for all parts of speech. This is expected since duluth3 is an ensemble approach that includes duluth2 as one of its members. The same relationship exists between duluth7 and duluth8 in the Spanish lexical sample, and comparable behavior is seen in Table 3 .

A more surprising case is the even higher level of agreement between the most common sense baseline and the lesk corpus baseline shown in Table 2 . This is not necessarily expected, and suggests that lesk corpus may not be finding a significant number of matches between the Senseval contexts and the WordNet glosses (as the lesk algorithm would hope to do) but instead may be relying on a simple default in many cases.

In previous work (Pedersen, 2001) we propose a 50-25-25 rule that suggests that about half of the instances in a supervised word sense disambiguation evaluation will be fairly easy for most systems to resolve, another quarter will be harder but possible for at least some systems, and that the final quarter will be very difficult for any system to resolve. 
Table 2: Pairwise Agreement English

\begin{tabular}{|c|c|c|}
\hline system pair & both-one-zero & kappa \\
\hline \multicolumn{3}{|c|}{ Nouns } \\
\hline common lesk_corp & 0.490 .060 .44 & 0.87 \\
\hline duluth2 duluth3 & 0.600 .080 .32 & 0.82 \\
\hline lesk_corp umcp & 0.530 .110 .36 & 0.78 \\
\hline duluth2 duluthB & 0.540 .140 .32 & 0.70 \\
\hline iit1 iit2 & 0.240 .130 .63 & 0.69 \\
\hline duluth3 duluthB & 0.560 .150 .29 & 0.68 \\
\hline common umcp & 0.480 .160 .36 & 0.68 \\
\hline ehu_all umcp & 0.550 .170 .29 & 0.64 \\
\hline uned_ls_t uned_ls_u & 0.410 .190 .40 & 0.63 \\
\hline duluth3 duluth4 & 0.550 .180 .27 & 0.61 \\
\hline duluth2 duluth4 & 0.520 .190 .29 & 0.60 \\
\hline duluth4 duluthB & 0.510 .190 .29 & 0.59 \\
\hline ehu_all lesk_corp & 0.500 .200 .30 & 0.59 \\
\hline cs224n duluth3 & 0.580 .190 .24 & 0.58 \\
\hline cs $224 n$ duluth 4 & 0.550 .190 .25 & 0.58 \\
\hline \multicolumn{3}{|c|}{ Verbs } \\
\hline common lesk_corp & 0.390 .060 .55 & 0.88 \\
\hline duluth2 duluth3 & 0.430 .070 .50 & 0.85 \\
\hline duluth3 duluth4 & 0.390 .140 .47 & 0.72 \\
\hline duluth2 duluth4 & 0.380 .150 .47 & 0.69 \\
\hline lesk_corp umcp & 0.380 .170 .44 & 0.65 \\
\hline common umcp & 0.360 .180 .46 & 0.65 \\
\hline cs224n duluth3 & 0.400 .200 .40 & 0.60 \\
\hline cs224n duluth4 & 0.380 .210 .41 & 0.59 \\
\hline cs224n duluth2 & 0.380 .210 .40 & 0.57 \\
\hline uned_ls_t uned_ls_u & 0.240 .200 .55 & 0.56 \\
\hline duluth3 lia & 0.390 .230 .38 & 0.54 \\
\hline lesk_corp talp & 0.360 .230 .40 & 0.54 \\
\hline cs224n lia & 0.410 .240 .35 & 0.53 \\
\hline common talp & 0.340 .240 .42 & 0.52 \\
\hline kunlp talp & 0.420 .240 .33 & 0.51 \\
\hline \multicolumn{3}{|c|}{ Adjectives } \\
\hline common lesk_corp & 0.590 .000 .41 & 0.99 \\
\hline duluth2 duluth3 & 0.630 .030 .34 & 0.93 \\
\hline lesk_corp umcp & 0.580 .070 .35 & 0.86 \\
\hline duluth2 duluthB & 0.590 .070 .35 & 0.86 \\
\hline duluth3 duluthB & 0.600 .070 .34 & 0.86 \\
\hline common umcp & 0.580 .070 .35 & 0.86 \\
\hline duluth4 duluthB & 0.550 .140 .32 & 0.71 \\
\hline duluth3 duluth4 & 0.570 .140 .30 & 0.71 \\
\hline cs224n duluth3 & 0.600 .130 .27 & 0.70 \\
\hline cs224n duluth2 & 0.590 .140 .27 & 0.70 \\
\hline
\end{tabular}

Table 3: Pairwise Agreement Spanish

\begin{tabular}{|c|c|c|}
\hline system pair & both-one-zero & kappa \\
\hline \multicolumn{3}{|c|}{ Nouns } \\
\hline duluth7 duluth8 & 0.570 .110 .32 & 0.76 \\
\hline umcp duluth9 & 0.500 .170 .33 & 0.65 \\
\hline duluth7 duluthY & 0.490 .210 .30 & 0.57 \\
\hline umcp duluthY & 0.480 .210 .31 & 0.56 \\
\hline duluth8 duluthY & $\begin{array}{llll}0.50 & 0.21 & 0.29\end{array}$ & 0.56 \\
\hline umcp duluth8 & 0.500 .230 .27 & 0.51 \\
\hline umcp duluth7 & 0.500 .230 .27 & 0.51 \\
\hline cs224 umcp & 0.510 .240 .25 & 0.49 \\
\hline duluth9 duluthY & 0.440 .260 .30 & 0.47 \\
\hline duluth8 duluth9 & 0.470 .270 .27 & 0.45 \\
\hline cs224 duluth9 & 0.470 .270 .26 & 0.44 \\
\hline cs224 jhu & 0.550 .250 .20 & 0.43 \\
\hline cs224 duluth8 & $\begin{array}{lll}0.51 & 0.27 & 0.22\end{array}$ & 0.42 \\
\hline jhu umcp & 0.510 .290 .21 & 0.38 \\
\hline jhu duluth8 & 0.530 .280 .19 & 0.37 \\
\hline \multicolumn{3}{|c|}{ Verbs } \\
\hline duluth7 duluth8 & 0.480 .080 .44 & 0.84 \\
\hline duluth8 duluth9 & 0.440 .140 .42 & 0.72 \\
\hline umcp duluth8 & 0.480 .140 .37 & 0.71 \\
\hline umcp duluth9 & 0.460 .160 .38 & 0.69 \\
\hline duluth7 duluth9 & 0.420 .160 .42 & 0.68 \\
\hline umcp duluth7 & 0.470 .160 .37 & 0.68 \\
\hline duluth8 duluthY & 0.440 .160 .39 & 0.67 \\
\hline duluth9 duluthY & 0.420 .180 .40 & 0.65 \\
\hline duluth7 duluthY & 0.430 .180 .39 & 0.64 \\
\hline umcp duluthY & 0.460 .190 .35 & 0.61 \\
\hline cs224 umcp & 0.490 .190 .32 & 0.61 \\
\hline cs224 duluth8 & 0.440 .250 .32 & 0.50 \\
\hline alicante umcp & $\begin{array}{lll}0.42 & 0.25 & 0.33\end{array}$ & 0.50 \\
\hline cs224 duluth7 & 0.430 .260 .32 & 0.48 \\
\hline cs224 jhu & 0.490 .250 .26 & 0.48 \\
\hline \multicolumn{3}{|c|}{ Adjectives } \\
\hline duluth7 duluth8 & 0.690 .060 .25 & 0.85 \\
\hline duluth7 duluthY & 0.600 .140 .26 & 0.68 \\
\hline umcp duluthY & 0.600 .150 .26 & 0.67 \\
\hline umcp duluth9 & 0.610 .140 .25 & 0.67 \\
\hline duluth8 duluthY & $\begin{array}{lll}0.61 & 0.15 & 0.24\end{array}$ & 0.67 \\
\hline umcp duluth8 & 0.640 .150 .21 & 0.64 \\
\hline duluth9 duluthY & 0.560 .180 .26 & 0.60 \\
\hline duluth8 duluth9 & 0.610 .170 .22 & 0.59 \\
\hline umcp duluth7 & 0.620 .170 .21 & 0.59 \\
\hline duluth7 duluth9 & 0.580 .200 .22 & 0.54 \\
\hline
\end{tabular}


This same idea could also be expressed by stating that the kappa agreement between two word sense disambiguation systems will likely be around 0.50 . In fact this is a common result in the full set of pairwise comparisons, particularly for overall results not broken down by part of speech. Tables 2 and 3 only list the largest kappa values, but even there kappa quickly reduces towards 0.50 . These same tables show that it is rare for two systems to agree on more than $60 \%$ of the correctly disambiguated instances.

\section{Optimal Combination}

An optimal combination is the accuracy that could be attained by a hypothetical tool called an optimal combiner that accepts as input the sense assignments for a test instance as generated by several different systems. It is able to select the correct sense from these inputs, and will only be wrong when none of the sense assignments is the correct one. Thus, the percentage accuracy of an optimal combiner is equal to one minus the percentage of instances that no system can resolve correctly.

Of course this is only a tool for thought experiments and is not a practical algorithm. An optimal combiner can establish an upper bound on the accuracy that could reasonably be attained over a particular sample of test instances.

Tables 4 and 5 list the top system pairs ranked by optimal combination (1.00 - value in zero column) for the English and Spanish lexical samples. Kappa scores are also shown to illustrate the interaction between agreement and optimal combination. Optimal combination is maximized when the percentage of instances where both systems are wrong is minimized. Kappa agreement is maximized by minimizing the percentage of instances where one or the other system (but not both) is correct. Thus, the only way a system pair could have a high measure of kappa and a high measure of optimal combination is if they were very accurate systems that disambiguated many of the same test instances correctly.

System pairs with low measures of agreement are potentially quite interesting because they are the most likely to make complementary errors. For example, in Table 5 under nouns, the alicante system has a low level of agreement with all of the other
Table 4: Optimal Combination English

\begin{tabular}{|c|c|c|}
\hline system pair & both-one-zero & kappa \\
\hline \multicolumn{3}{|c|}{ Nouns } \\
\hline kunlp smuls & 0.490 .390 .12 & 0.11 \\
\hline smuls talp & 0.480 .390 .13 & 0.11 \\
\hline cs224n kunlp & 0.480 .390 .13 & 0.11 \\
\hline ehu_all smuls & 0.470 .400 .13 & 0.10 \\
\hline $\operatorname{cs} 224 n$ talp & 0.480 .390 .14 & 0.13 \\
\hline jhu_final kunlp & 0.490 .370 .14 & 0.16 \\
\hline smuls umcp & 0.450 .410 .14 & 0.10 \\
\hline kunlp lia & 0.480 .380 .14 & 0.14 \\
\hline lia talp & 0.470 .390 .14 & 0.13 \\
\hline jhu_final talp & 0.480 .380 .14 & 0.15 \\
\hline duluth3 kunlp & 0.470 .380 .15 & 0.15 \\
\hline cs224n ehu_all & 0.480 .380 .15 & 0.16 \\
\hline ehu_all lia & 0.470 .380 .15 & 0.15 \\
\hline ehu_all jhu_final & 0.480 .360 .16 & 0.19 \\
\hline duluth3 talp & 0.470 .380 .16 & 0.17 \\
\hline \multicolumn{3}{|c|}{ Verbs } \\
\hline jhu_final kunlp & 0.340 .460 .20 & 0.06 \\
\hline ehu_all jhu_final & 0.310 .440 .21 & 0.07 \\
\hline ehu_all smuls & 0.310 .440 .21 & 0.07 \\
\hline ehu_all kunlp & $\begin{array}{llll}0.33 & 0.41 & 0.22\end{array}$ & 0.13 \\
\hline kunlp smuls & 0.360 .430 .22 & 0.13 \\
\hline cs224n ehu_all & 0.290 .450 .22 & 0.05 \\
\hline ehu_all lia & 0.300 .440 .22 & 0.08 \\
\hline cs224n kunlp & 0.330 .440 .23 & 0.11 \\
\hline alicante ehu_all & 0.260 .470 .23 & 0.03 \\
\hline kunlp lia & 0.340 .420 .23 & 0.14 \\
\hline jhu_final talp & 0.320 .440 .24 & 0.12 \\
\hline duluth3 ehu_all & 0.260 .460 .24 & 0.05 \\
\hline ehu_all talp & $\begin{array}{llll}0.30 & 0.41 & 0.24\end{array}$ & 0.13 \\
\hline alicante jhu_final & 0.300 .450 .24 & 0.09 \\
\hline jhu_final umcp & 0.300 .450 .24 & 0.09 \\
\hline \multicolumn{3}{|c|}{ Adjectives } \\
\hline alicante jhu_final & 0.460 .370 .08 & 0.03 \\
\hline alicante smuls & 0.410 .410 .09 & -0.04 \\
\hline alicante cs $224 n$ & 0.420 .400 .09 & -0.01 \\
\hline alicante kunlp & 0.410 .390 .11 & 0.03 \\
\hline alicante lia & 0.410 .390 .11 & 0.03 \\
\hline alicante duluth3 & 0.400 .400 .11 & 0.02 \\
\hline alicante talp & 0.400 .410 .11 & 0.02 \\
\hline alicante ehu_all & 0.410 .390 .11 & 0.05 \\
\hline alicante umcp & 0.390 .400 .12 & 0.04 \\
\hline alicante duluth2 & 0.390 .400 .12 & 0.03 \\
\hline
\end{tabular}


Table 5: Optimal Combination Spanish

\begin{tabular}{|c|c|c|}
\hline system pair & both-one-zero & kappa \\
\hline \multicolumn{3}{|c|}{ Nouns } \\
\hline alicante jhu & 0.290 .320 .11 & 0.06 \\
\hline alicante duluth7 & 0.270 .340 .12 & 0.03 \\
\hline alicante duluthY & 0.250 .350 .12 & 0.01 \\
\hline alicante duluth8 & 0.280 .320 .13 & 0.08 \\
\hline alicante cs224 & 0.280 .320 .13 & 0.09 \\
\hline alicante umcp & 0.260 .330 .14 & 0.06 \\
\hline alicante duluth9 & 0.260 .310 .16 & 0.14 \\
\hline jhu duluthY & 0.460 .360 .18 & 0.24 \\
\hline jhu duluth7 & 0.510 .290 .19 & 0.35 \\
\hline jhu duluth8 & 0.530 .280 .19 & 0.37 \\
\hline cs224 jhu & 0.550 .250 .20 & 0.43 \\
\hline jhu duluth9 & 0.460 .340 .20 & 0.29 \\
\hline jhu umcp & $\begin{array}{lll}0.51 & 0.29 & 0.21\end{array}$ & 0.38 \\
\hline $\operatorname{cs} 224$ duluth7 & 0.490 .300 .22 & 0.36 \\
\hline cs224 duluth8 & 0.510 .270 .22 & 0.42 \\
\hline \multicolumn{3}{|c|}{ Verbs } \\
\hline jhu duluthY & 0.390 .380 .23 & 0.23 \\
\hline jhu umcp & 0.480 .270 .25 & 0.44 \\
\hline jhu duluth9 & 0.390 .360 .26 & 0.29 \\
\hline jhu duluth8 & 0.420 .320 .26 & 0.35 \\
\hline cs224 jhu & 0.490 .250 .26 & 0.48 \\
\hline jhu duluth7 & 0.420 .320 .26 & 0.36 \\
\hline alicante jhu & 0.450 .260 .29 & 0.47 \\
\hline $\operatorname{cs} 224$ duluthY & 0.430 .270 .30 & 0.46 \\
\hline alicante cs224 & $\begin{array}{lll}0.41 & 0.28 & 0.31\end{array}$ & 0.44 \\
\hline alicante duluthY & 0.350 .340 .31 & 0.32 \\
\hline cs224 umcp & 0.490 .190 .32 & 0.61 \\
\hline cs 224 duluth7 & 0.430 .260 .32 & 0.48 \\
\hline cs224 duluth8 & 0.440 .250 .32 & 0.50 \\
\hline cs224 duluth9 & 0.410 .270 .32 & 0.47 \\
\hline alicante umcp & 0.420 .250 .33 & 0.50 \\
\hline \multicolumn{3}{|c|}{ Adjectives } \\
\hline jhu duluth8 & 0.660 .220 .12 & 0.39 \\
\hline jhu duluth7 & 0.640 .240 .12 & 0.36 \\
\hline jhu duluthY & $\begin{array}{llll}0.56 & 0.31 & 0.12\end{array}$ & 0.25 \\
\hline alicante jhu & 0.620 .260 .13 & 0.33 \\
\hline jhu duluth9 & 0.590 .290 .13 & 0.29 \\
\hline cs224 jhu & 0.700 .160 .13 & 0.51 \\
\hline jhu umcp & 0.640 .230 .13 & 0.38 \\
\hline alicante cs 224 & $\begin{array}{lll}0.61 & 0.24 & 0.15\end{array}$ & 0.39 \\
\hline cs224 duluth8 & 0.660 .190 .16 & 0.50 \\
\hline $\operatorname{cs} 224$ duluth7 & 0.640 .200 .16 & 0.49 \\
\hline
\end{tabular}

Table 6: Difficulty of Instances

\begin{tabular}{rrrrr}
\hline \hline$\#$ & \multicolumn{1}{c}{ noun } & \multicolumn{1}{c}{ verb } & \multicolumn{1}{c}{ adj } & \multicolumn{1}{c}{ total } \\
\hline & $59(16)$ & $174(6)$ & $29(8)$ & $262(8)$ \\
1 & $51(15)$ & $116(10)$ & $26(14)$ & $193(12)$ \\
2 & $59(18)$ & $122(12)$ & $41(21)$ & $222(15)$ \\
3 & $64(19)$ & $117(16)$ & $29(23)$ & $210(18)$ \\
4 & $84(17)$ & $102(16)$ & $28(18)$ & $214(17)$ \\
5 & $76(23)$ & $76(18)$ & $24(20)$ & $176(21)$ \\
6 & $53(28)$ & $61(30)$ & $23(31)$ & $137(29)$ \\
7 & $51(29)$ & $65(22)$ & $23(34)$ & $139(27)$ \\
8 & $62(27)$ & $58(34)$ & $18(31)$ & $138(30)$ \\
9 & $47(32)$ & $69(28)$ & $17(26)$ & $133(29)$ \\
10 & $62(28)$ & $61(32)$ & $18(30)$ & $141(30)$ \\
11 & $55(39)$ & $56(26)$ & $21(38)$ & $132(34)$ \\
12 & $80(40)$ & $61(41)$ & $22(35)$ & $163(40)$ \\
13 & $86(58)$ & $56(34)$ & $21(45)$ & $163(48)$ \\
14 & $125(65)$ & $62(49)$ & $33(51)$ & $220(59)$ \\
15 & $131(77)$ & $125(99)$ & $36(60)$ & $292(84)$ \\
16 & $141(83)$ & $107(117)$ & $61(70)$ & $309(92)$ \\
17 & $133(75)$ & $100(162)$ & $86(74)$ & $319(101)$ \\
18 & $92(73)$ & $80(203)$ & $102(80)$ & $274(113)$ \\
19 & $97(68)$ & $59(170)$ & $49(77)$ & $205(100)$ \\
20 & $65(66)$ & $38(192)$ & $30(49)$ & $133(96)$ \\
21 & $42(68)$ & $15(155)$ & $17(47)$ & $74(79)$ \\
22 & $29(70)$ & $15(73)$ & $7(39)$ & $51(67)$ \\
23 & $10(49)$ & $11(52)$ & $7(38)$ & $28(47)$ \\
\hline & & Spanish & \\
0 & $50(16)$ & $126(12)$ & $52(24)$ & $228(16)$ \\
1 & $81(18)$ & $63(17)$ & $32(36)$ & $176(21)$ \\
2 & $63(24)$ & $69(18)$ & $42(50)$ & $174(28)$ \\
3 & $63(27)$ & $55(23)$ & $39(81)$ & $157(39)$ \\
4 & $74(32)$ & $47(23)$ & $43(101)$ & $164(47)$ \\
5 & $94(35)$ & $49(28)$ & $35(77)$ & $178(42)$ \\
6 & $87(40)$ & $61(39)$ & $57(90)$ & $205(53)$ \\
7 & $182(47)$ & $94(46)$ & $88(93)$ & $364(58)$ \\
8 & $105(44)$ & $181(62)$ & $293(166)$ & $579(111)$
\end{tabular}


Table 8: Difficulty of Spanish Word Types

\begin{tabular}{lr|lr}
\hline \hline word-pos (test) & \multicolumn{1}{r}{ mean } & word-pos (test) & mean \\
\hline claro-a (66) & 7.6 & verde-a (33) & 5.3 \\
local-a (55) & 7.4 & canal-n (41) & 5.3 \\
popular-a (204) & 7.1 & clavar-v (44) & 5.1 \\
partido-n (57) & 7.0 & masa-n (41) & 5.1 \\
bomba-n (37) & 6.8 & apuntar-v (49) & 4.9 \\
brillante-a (87) & 6.7 & autoridad-n (34) & 4.9 \\
usar-v (56) & 6.5 & tocar-v (74) & 4.8 \\
tabla-n (41) & 6.3 & explotar-v (41) & 4.7 \\
vencer-v (65) & 6.3 & programa-n (47) & 4.7 \\
simple-a (57) & 6.2 & circuito-n (49) & 4.3 \\
hermano-n (57) & 6.1 & copiar-v (53) & 4.3 \\
apoyar-v (73) & 6.0 & actuar-v (55) & 4.2 \\
vital-a (79) & 5.9 & operacion-n (47) & 4.2 \\
gracia-n (61) & 5.9 & pasaje-n (41) & 4.1 \\
organo-n (81) & 5.8 & saltar-v (37) & 4.1 \\
corona-n (40) & 5.5 & tratar-v (70) & 3.9 \\
ciego-a (42) & 5.5 & natural-a (58) & 3.9 \\
corazon-n (47) & 5.5 & grano-n (22) & 3.9 \\
coronar-v (74) & 5.4 & conducir-v (54) & 3.8 \\
naturaleza-n (56) & 5.4 & &
\end{tabular}

green-a (94)

fatigue-n (43)

oblique-a (29)

nation-n (37)

church-n (64)

local-a (38)

fit-a (29)

use-v (76)

child-n (64)

wander-v (50)

begin-v (280)

bum-n (45)

feeling-n (51)

facility-n (58)

colorless (35)

grip-n (51)

day-n (145)

mouth-n (60)

material-n (69)

art-n (98)

ty of English Word Types

mean word-pos (test) mean

20.2 circuit-n (85)

18.3 sense-n (53)

17.7 authority-n (92)

17.5 replace-v (45)

17.3 restraint-n (45)

16.7 live-v (67)

16.5 treat-v (44)

16.5 free-a (82)

16.1 nature-n (46)

16.0 simple-a (66)

16.0 dress-v (59)

15.7 cool-a (52)

15.5 bar-n (151)

15.3 stress-n (39)

15.1 channel-n (73)

15.1 match-v (42)

14.9 natural-a (103)

14.9 serve-v (51)

14.3 train-v (63)

14.0 post-n (79)

13.8 fine-a (70)

13.6 drift-v (32)

13.4 leave-v (66)

13.4 play-v (66)

13.0 wash-v (12)

12.9 keep-v (67)

12.6 work-v (60)

12.5 drive-v (42)

11.4 develop-v (69)

11.1 carry-v (66)

11.1 see-v (69)

11.1 strike-v (54)

11.0 call-v (66)

11.0 pull-v (60)

11.0 turn-v (67)

10.7 draw-v (41)

find-v (68)

10.7

10.6

10.5

10.4

10.3

10.2

10.1

10.0

10.0

9.8

9.7

9.7

9.5

9.5

9.2

9.0

9.0

8.8

5.9

5.7
8.7 systems. However, the measure of optimal combi-

8.7 nation is quite high, reaching $0.89(1.00-0.11)$ for

8.6 the pair of alicante and jhu. In fact, all seven of the

7.7 other systems achieve their highest optimal combi-

7.7 nation value when paired with alicante.

7.5 This combination of circumstances suggests that

7.4 the alicante system is fundamentally different than

7.4 the other systems, and is able to disambiguate a cer-

7.0 tain set of instances where the other systems fail. In

6.8 fact the alicante system is different in that it is the

6.6 only Spanish lexical sample system that makes use

6.3 of the structure of Euro-WordNet, the source of the

6.3 sense inventory.

\subsection{Instance Difficulty}

5.0 The difficulty of disambiguating word senses can

4.7 vary considerably. A word with multiple closely re-

4.2 lated senses is likely to be more difficult than one with a few starkly drawn differences. In supervised learning, a particular sense of a word can be difficult to disambiguate if there are a small number of training examples available.

Table 6 shows the distribution of the number of 
instances that are successfully disambiguated by a particular number of systems in both the English and Spanish lexical samples. The value under the \# column shows the number of systems that are able to disambiguate the number of noun, verb, adjective and total instances shown in the row. The average number of training examples available for the correct answers associated with these instances is shown in parenthesis. For example, the first line shows that there were 59 noun instances that no system (of 23) could disambiguate, and that there were on average 16 training examples available for each of the correct senses for these 59 instances.

Two very clear trends emerge. First, there are a substantial number of instances that are not disambiguated correctly by any system (262 in English, 228 in Spanish) and there are a large number of instances that are disambiguated by just a handful of systems. In the English lexical sample, there are 1,277 test instances that are correctly disambiguated by five or fewer of the 23 systems. This is nearly $30 \%$ of the test data, and confirms that this was a very challenging set of test instances.

There is also a very clear correlation between the number of training examples available for a particular sense of a word and the number of systems that are able to disambiguate instances of that word correctly. For example, Table 6 shows that there were 174 English verb instances that no system disambiguated correctly. On average there were only 6 training examples for the correct senses of these instances. However, there were 28 instances that all 23 English systems were able to disambiguate. For these instances an average of 47 training examples were available for each correct sense.

This correlation between instance difficulty and number of training examples may suggest that future SENSEVAL exercises provide a minimum number of training examples for each sense, or adjust the scoring to reflect the difficulty of disambiguating a sense with very few training examples.

Finally, we assess the difficulty associated with word types by calculating the average number of systems that were able to disambiguate the instances associated with that type. This information is provided for the English and Spanish lexical samples in Tables 7 and 8. Each word is shown with its part of speech, the number of test instances, and the average number of systems that were able to disambiguate each of the test instances.

The verb collaborate is the easiest according to this metric in the English lexical sample. It has 30 test instances that were disambiguated correctly by an average of 20.2 of the 23 systems. The verb find proves to be the most difficult, with 68 test instances disambiguated correctly by an average of 4.2 systems. A somewhat less extreme range of values is observed for the Spanish lexical sample in Table 8. The adjective claro had 66 test instances that were disambiguated correctly by an average of 7.6 of the 8 systems. The most difficult word was the verb conducir, which has 54 test instances that were disambiguated correctly by an average of 3.8 systems.

\section{Conclusion}

This paper presents an analysis of the results from the English and Spanish lexical sample tasks of SENSEVAL-2. The analysis is based on the kappa statistic and a measure known as optimal combination. It also assesses the difficulty of the test instances in these lexical samples. We find that there are a significant number of test instances that were not disambiguated correctly by any system, and that there is some correlation between instance difficulty and the number of available training examples.

\section{Acknowledgments}

This work has been partially supported by a National Science Foundation Faculty Early CAREER Development award (\#0092784).

\section{References}

J. Carletta. 1996. Assessing agreement on classification tasks: the kappa statistic. Computational Linguistics, 22(2).

J. Cohen. 1960. A coefficient of agreement for nominal scales. Educ. Psychol. Meas., 20:37-46.

K. Krippendorf. 1980. Content Analysis: An Introduction to its Methodology. Sage Publications, Thousand Oaks, CA.

T. Pedersen. 2001. Machine learning with lexical features: The Duluth approach to SENSEVAL-2. In Proceedings of the Senseval-2 Workshop, Toulouse, July. 